\title{
Influencia de la Carga Organizacional y Trastornos del Sueño en la Accidentabilidad de Conductores de Camiones
}

\author{
INFLUENCE OF BOTH THE ORGANIZATIONAL BURDEN AND SLEEP DISORDERS IN ACCIDENT RATES OF \\ TRUCK DRIVERS
}

\author{
Eduardo Navarrete Espinoza', Natali Feliu Saavedra², Gabriela Bahamondes Valenzuela ${ }^{3}$ \\ 1. Académico e Investigador del Grupo GESE, Escuela de Ciencias y Tecnologias, Campus Los Ángeles, Universidad de Concepción, Los Ángeles, Chile. \\ 2. Ingeniero en Prevención de Riesgos. Profesional independiente, Los Ángeles, Chile. \\ 3. Psicólogo organizacional, Campus Los Ángeles, Universidad de Concepción, Los Ángeles, Chile.
}

\section{RESUMEN}

Con el objetivo de determinar la influencia de los niveles de estrés, trastornos del sueño y carga organizacional en la accidentabilidad de conductores que trabajan en diferentes sistemas de turnos se realizó un estudio en una empresa de transporte forestal. Las variables estudiadas fueron: estrés, trastornos del sueño (insomnio, somnolencia, apnea del sueño y síndrome de piernas inquietas), factores de riesgo psicosocial, impacto de los turnos y accidentabilidad. Como resultado se determinó que el turno rotativo presentó mayor cantidad de conductores con presencia de trastornos del sueño que el turno diurno. La evaluación de riesgos psicosociales clasificó al turno diurno con riesgo alto nivel $1 \mathrm{y}$ al turno rotativo sin riesgo. A modo de conclusión se determinó que existe relación entre accidentabilidad y las variables evaluadas, presentándose correlaciones significativas para el turno diurno, entre accidentabilidad, estrés y apoyo social; en cambio, el turno rotativo, presentó una correlación significativa entre accidentabilidad y compensaciones.

(Navarrete E, Feliu N, Bahamondes G, 2017. Influencia de la Carga Organizacional y Trastornos del Sueño en la Accidentabilidad de Conductores de Camiones. Cienc Trab. May-Ago; 19 [59]: 67-75).

Palabras clave: INFLUENCIA DE LOS TURNOS, CARGA ORGANIZACIONAL, TRASTORNOS DEL SUEÑO, ESTRÉS, TRANSPORTE FORESTAL.

\section{ABSTRACT}

A study was conducted in a transport company, in order to determine the influence of organizational burden and sleep disorders in both rotating shift and daytime shifts of truck drivers. The studied variables were: stress, sleep disorders (insomnia, drowsiness, sleep apnea and restless leg syndrome), psychosocial risk factors, impact of working shifts and accidents. It found more frequency of drivers with sleep disorder in rotating shift than daytime shift. The daytime shift was describe as high psychosocial risk, level 1, and rotating shift was describe as without risk according to the same evaluation. The psychosocial risk of daytime shift was evaluated as high risk, level 1, and rotating shift was described as without risk. A relationship among accident rates and the studied variables was determined. The daytime shift showed significant correlations among variables such as accident rates, stress and social support; in the other hand, the correlation among accident rates and compensation was significant to the rotating shift.

Keywords: WORKING SHIFTS INFLUENCE, ORGANIZATIONAL BURDEN, SLEEP DISORDERS, STRESS, AND FOREST TRANSPORT.

\section{INTRODUCCIÓN}

En Chile existe un total de 35.625 empresas cuya actividad económica es el transporte de carga por carretera, siendo los sectores con mayor participación el de construcción, con un $26 \%$ y forestal con un 20\%. ${ }^{1}$ Un estudio de la Universidad de Génova, Italia, realizado el año 2011, determinó que un 3,2\% del total de

\section{Correspondencia / Correspondence:}

Eduardo Navarrete Espinoza

Universidad de Concepción

Escuela de Ciencias y Tecnologías, Campus Los Ángeles

Juan Antonio Coloma 0201, Los Ángeles, Chile

Tel. (56-43) 2405217

e-mail: ednavarr@udec.cl

Recibido: 27 de Enero 2017 /Aceptado: 28 de Marzo 2017 accidentes ocurridos en ese periodo se atribuyeron a dormirse al volante y a somnolencia. La información recopilada apuntó a la alta peligrosidad y mortalidad de los accidentes relacionados con sueño; también se demostró la existencia de una relación con los efectos circadianos, concluyendo que la mayoría de los accidentes se producen entre las 2 y 6 de la madrugada. ${ }^{2}$ Los registros de la Comisión Nacional de Seguridad de Tránsito [CONASET] ${ }^{3}$, emplazan a los usuarios de camiones, con un total de 5.979 accidentes, 78 personas fallecidas y 1.539 lesionados. De acuerdo a los análisis realizados por CONASET ${ }^{3}$, los accidentes se clasifican, a nivel general, según las siguientes causas: alcohol en conductor, pérdida de control de vehículo, desobediencia a señalización, imprudencia del conductor y otras causas.

Es importante considerar dentro de las causas que generan accidentes, la disminución de la concentración durante la ejecución de una tarea en un turno nocturno, debido a la somnolencia producida en ese momento de la jornada, ya que al disminuir la eficiencia en el trabajo, aumenta la posibilidad de errores y de 
accidentes. ${ }^{4}$ En Chile, cerca del 25\% de la población laboral dependiente trabaja en algún sistema de turnos; siendo creciente además, la aplicación de este esquema de organización del trabajo en los distintos sectores industriales, incluyendo el de servicios. ${ }^{5} \mathrm{El}$ trabajo en turnos se define como aquel que se realiza en horarios alternados de la jornada o bien en forma permanente en horarios inusuales del día. Los turnos rotativos son un esquema horario donde los trabajadores pueden realizar su actividad en la mañana, tarde o noche, en una secuencia determinada; y los turnos fijos, aquellos donde en forma permanente se trabaja en un horario determinado, sin posibilidad de cambiar el turno. ${ }^{6}$

Los seres humanos poseen ritmos biológicos generados por osciladores internos que forman parte del patrimonio genético; estos ritmos pueden ser alterados por factores exógenos como el ciclo de luz-oscuridad, entorno social y, por supuesto, horario de trabajo. Ocurre una alteración de los ritmos circadianos cuando entre ellos se presenta una desorganización, o bien, cuando ocurre una desincronización entre los ritmos generados por el reloj biológico y el ciclo ambiental de 24 horas. ${ }^{6}$ Es posible la adaptación al trabajo nocturno, aunque lentamente. No obstante, el organismo humano está mejor preparado para mantenerse alerta y activo durante el día; en consecuencia, el ajuste al trabajo nocturno nunca sería completo, pues se perdería durante los días libres. ${ }^{7}$ El trabajo en turnos ha sido reconocido como un factor de riesgo ocupacional; la evidencia científica indica que esta forma de organización del trabajo puede representar una condición de tensión biológica, mental y social, que comprometería la salud, seguridad, rendimiento y bienestar de los trabajadores. ${ }^{8}$ Habitualmente, se ocupan 3 categorías para clasificar el impacto de los turnos en las personas: trastornos del sueño, alteraciones físicas y psicológicas, y trastornos sociales y familiares. ${ }^{9-11}$ Con respecto a los efectos sobre el sueño, éste se ve afectado tanto en calidad como en cantidad, siendo una de las principales funciones que se alteran con el trabajo en turnos. El sueño que se logra en el día, es dos o tres horas más corto que el nocturno; asimismo, es más ligero y vulnerable a los estímulos ambientales, como el ruido. Este problema se agudiza con los años, pues el sueño se hace cada vez más frágil y corto con la edad. ${ }^{12}$ El rendimiento y seguridad también se ven afectados, ya que los ritmos circadianos influyen en el nivel de alerta de las personas; el rendimiento humano es más alto en el período de vigilia diurna, con un nadir a primera hora de la mañana y luego, otra disminución menos acentuada al iniciar la tarde, esto traería como consecuencia una mayor probabilidad de cometer errores en el turno nocturno. ${ }^{13}$ Los trastornos del sueño se definen como una patología muy frecuente, tanto aislada, propia o asociada a otros trastornos, siendo los trabajadores más expuestos a este tipo de patologías aquellos que cuentan con un turno rotativo (día-noche). Los trastornos del sueño a los cuales se pueden ver enfrentados son: Insomnio, que es la dificultad para conciliar o mantener el sueño. Las jornadas o turnos laborales que se solapan con el período normal del sueño obligan al trabajador a permanecer alerta en un momento inadecuado de su ciclo sueño-vigilia. No solo se presenta en trabajadores que tienen turnos nocturnos, sino también en aquellos con rotación de turnos, ya que estos últimos necesitan un tiempo determinado para adaptar su ciclo sueño-vigilia a la nueva situación. ${ }^{14}$ En Chile, el $25 \%$ de la población presenta insomnio crónico. ${ }^{15}$ Somnolencia, son episodios de sueño que están presentes solo cuando se requieren tiempos de descanso o cuando existe poca atención, como puede suceder en situaciones cotidianas. Apnea del sueño, son episodios repetitivos de obstrucción de la vía aérea superior que ocurren durante el sueño. Según Olivi ${ }^{16}$, el 5\% de la población chilena adulta sufre de apnea. Síndrome de piernas inquietas, es un trastorno caracterizado por sensaciones desagradables en las piernas, impidiendo a las personas dejar de moverlas. ${ }^{17}$ Los trabajadores que se desempeñan en turnos rotativos (dia-noche) y que tienen que dormir de día, dependiendo del turno, deben enfrentar una serie de obstáculos, como lo son, el horario en que el resto de las personas realizan tareas cotidianas, la gran intensidad de luz y ruido durante el día, además de las altas temperaturas, dependiendo la estación del año. ${ }^{4}$ La luz tiene un efecto supresor sobre la secreción de melatonina; en el caso de los trabajadores en turno nocturno, activa el organismo cuando al trabajador le corresponde descansar y lo relaja cuando debe estar activo. ${ }^{18}$ Para obtener un sueño óptimo, reparador o de buena calidad, el tiempo dedicado al mismo debería coincidir con el ritmo circadiano biológico del sueño de cada individuo. ${ }^{14} \mathrm{El}$ grado de ajuste al trabajo por turnos depende de un gran número de variables, entre las que se encuentran la duración del turno, el número de turnos de noche en cada rotación, la dirección y la velocidad de la rotación; siendo tampoco ajenos los factores individuales como la edad o años de trabajo. Todo ello dificulta el carácter reponedor del sueño aumentando, como consecuencia, la probabilidad de verse afectado por problemas relacionados con la salud a medio y largo plazo. Los escasos estudios existentes muestran un impacto importante en la salud mental de las personas afectadas por el sistema de trabajo y los trastornos del sueño. ${ }^{4}$

Los atributos organizacionales que se componen de la distribución de los turnos y los factores psicosociales, tienen la capacidad de afectar, en forma positiva o negativa, el bienestar y la salud (física, psíquica o social) del trabajador. La carga organizacional evalúa dos áreas, el impacto del trabajo en turnos en las personas y los factores psicosociales. ${ }^{6}$ Cuando los factores psicosociales son disfuncionales, provocan respuestas de inadaptación, de tensión, respuestas psicofisiológicas de estrés, y pasan a ser factores psicosociales de riesgo o de estrés, afectando a la salud y al bienestar del trabajador. ${ }^{19}$ La exposición a un estresor, factor psicosocial y organizacional provoca una respuesta, y ésta, bajo ciertas circunstancias, puede tener consecuencias sobre la salud, la seguridad o el rendimiento. ${ }^{20}$ Desde una perspectiva laboral, son varios los factores identificados como estresores: el ambiente físico, las demandas del trabajo, los contenidos del trabajo, el desempeño de los roles, las relaciones interpersonales y el desarrollo de la carrera, las nuevas tecnologías y las fuentes extra-organizacionales, formando los turnos rotativos, parte de las demandas estresantes del trabajo. ${ }^{4}$ El estrés afecta de forma diferente a cada persona, algunas de las consecuencias que pueden generarse están asociadas a las alteraciones en el procesamiento de la información, que incluye efectos sobre la memoria, el tiempo de reacción, precisión, la atención y la toma de decisiones, estando estas consecuencias ligadas a las reacciones frente a algún tipo de accidente. ${ }^{6}$

Los trabajadores con sistema de rotación requieren una adaptación constante de un programa de turnos a otro (semana de día a semana de noche). De esta forma, el cuerpo se encuentra inmerso en un cambio continuo, por lo que se espera que se produzcan más problemas que en el caso de otros horarios más convencionales. ${ }^{4}$ Por lo anteriormente expuesto, es importante el estudio de la relación entre la accidentabilidad, trastornos del sueño, estrés y carga organizacional en conductores de camiones, tanto en turnos rotativos como en un solo turno, ya que los trastornos y los aspectos psicológicos 
mencionados con anterioridad, no son considerados en su totalidad como un factor importante en la generación de accidentes.

El objetivo general de este estudio fue determinar la influencia de los niveles de estrés, trastornos del sueño y carga organizacional en la accidentabilidad de conductores que trabajan en diferentes sistemas de turnos. Como objetivos específicos se propusieron: a) determinar los niveles de estrés y trastornos del sueño a los que se encuentran expuestos los conductores en turnos rotativos y diurno; b) determinar la presencia de factores de riesgo psicosocial en los conductores de distintos turnos; c) determinar el impacto del trabajo en turnos sobre los conductores de ambos turnos; d) identificar el tipo de turno que tiene mayor accidentabilidad; y, e) determinar la relación entre estrés, factores de riesgo psicosocial, trastornos del sueño, influencia de los turnos, y la accidentabilidad en los sistemas de turno bajo estudio.

\section{MATERIAL Y MÉTODOS}

La investigación se realizó bajo un diseño no experimental, de tipo transversal, descriptivo y correlacional, en una población de conductores de camiones forestales con turnos rotativos y diurnos.

\section{Muestra}

La muestra estuvo compuesta por 48 conductores, 30 correspondientes al turno rotativo y 18 al turno diurno; éstos cumplieron los siguientes criterios de inclusión:

- Pertenecer a turnos rotativos y diurnos.

- Las faenas en que se desarrollaban ambos turnos debían tener condiciones de trabajo similares (caminos, distancias de conducción, tipos de camión).

- Haber firmado el consentimiento informado.

\section{Variables de estudio e instrumentos de medición}

Para la evaluación de factores psicosociales, estrés y carga organizacional se utilizaron los siguientes tests psicométricos:

Estrés: La percepción de estrés se midió con la Escala de Estrés Percibido PSS-14 ${ }^{21}$, diseñada para medir el grado en que los individuos evalúan situaciones de su vida como estresantes. Los items incluidos permiten evaluar cuán impredecible, incontrolable y sobrecargada encuentran su vida las personas encuestadas. La PSS-14 incluye 14 preguntas con respuestas que varían entre 0 y 4 puntos para cada una. Puntajes finales elevados indican altos niveles de estrés. ${ }^{22}$ El nivel de confiabilidad de este cuestionario corresponde a un Alfa de Cronbach de 0,79 , lo que es aceptable. ${ }^{23}$

Factores de riesgo psicosocial: Para la evaluación de esta variable se utilizó el cuestionario ISTAS 21 versión breve. Este instrumento tiene por objetivo identificar la presencia y nivel de exposición a riesgos psicosociales al interior de una organización. Evalúa 5 dimensiones: a) exigencias psicológicas, b) trabajo activo y desarrollo de habilidades, c) apoyo social en la empresa y calidad de liderazgo, d) compensaciones y estima y e) doble presencia. Los resultados se muestran con un sistema gráfico, en que dentro de cada dimensión analizada se establece la prevalencia de la población en tres niveles de riesgo: verde, señala que el riesgo psicosocial es bajo (es la mejor condición para trabajar y se puede señalar como factor de protección); amarillo, señala una condición de riesgo moderado; y rojo, una condición de alto riesgo (la peor condición de trabajo). ${ }^{24}$

BESIAK: Esta metodología se utilizó para la evaluación del impacto de un esquema horario en los trabajadores. Contiene 14 indicadores que permiten obtener un puntaje total, que se encuentra en un rango de 0 a 1000 puntos. Según Gissel \& Knauth $^{25}$, puntajes totales mayores a 309,24 indicarian un incumplimiento creciente en recomendaciones de ergonomía, en cuanto al diseño del sistema de turno; es decir, existiría la presencia de efectos importantes sobre la salud, bienestar, adaptación y rendimiento de los trabajadores. La puntuación de referencia establece los siguientes rangos: a) riesgo bajo, puntaje total menor o igual a 300 puntos; b) riesgo medio, puntaje total entre 301 y 600 puntos y c) riesgo alto, puntaje mayor a 600 puntos.

Trastornos del sueño: Para la evaluación de los trastornos del sueño, se utilizó un cuestionario, el cual incluía insomnio, somnolencia, apnea del sueño y síndrome de piernas inquietas. ${ }^{17}$ Para el caso de insomnio, se utilizó el Insomnia Severity Index ${ }^{26}$; para somnolencia, la Escala de Epworth ${ }^{27}$; para el síndrome de piernas inquietas y apnea, el Pittsburg Sleep Quality Index ${ }^{28}$ y Cuestionario de Oviedo del Sueño. ${ }^{29}$

\section{Análisis estadístico}

Se realizó un análisis descriptivo de cada variable evaluada, obteniendo estadígrafos tanto de tendencia central, como de dispersión. La correlación de las variables cuantitativas se analizó por medio de la prueba no paramétrica de Spearman ${ }^{30,31}$, dado el no cumplimiento del supuesto de normalidad, el cual fue comprobado por medio Shapiro-Wilk. ${ }^{32}$ Los datos fueron analizados con el software Statistica 10.0, utilizando un nivel de significancia de 0,05.

\section{RESULTADOS}

\section{Características socio-demográficas y laborales de los participantes}

La edad de los trabajadores evaluados fluctuó entre 29 y 63 años (promedio 43,4 años), presentándose un valor medio de 45,6 años y 42,0 años para los turnos diurno y rotativo, respectivamente. El rango de edad con mayor frecuencia en ambos turnos correspondió a 41-60 años, representado por un 53,3\% y 50,0\% (turno rotativo y diurno, respectivamente). Respecto al estado civil, el $63,3 \%$ y $61,1 \%$ de los conductores de los turnos rotativo y diurno, respectivamente, se encontraba casado (Tabla 1).

Tabla 1.

Características de la muestra según tipo de turno.

\begin{tabular}{|c|c|c|c|c|c|c|}
\hline & \multicolumn{2}{|c|}{ Turno Rotativo } & \multicolumn{2}{|c|}{ Turno Diurno } & \multicolumn{2}{|c|}{ Total } \\
\hline & $\mathrm{N}$ & $\%$ & $\mathrm{~N}$ & $\%$ & $\mathrm{~N}$ & $\%$ \\
\hline Casos & 30,0 & 62,5 & 18,0 & 37,5 & 48,0 & 100,0 \\
\hline \multicolumn{7}{|c|}{ Distribución según rango de edad (años) } \\
\hline $20-40$ & 12,0 & 40,0 & 7,0 & 38,9 & 19,0 & 39,6 \\
\hline $41-60$ & 16,0 & 53,3 & 9,0 & 50,0 & 25,0 & 52,1 \\
\hline$>60$ & 0,0 & 0,0 & 2,0 & 11,1 & 2,0 & 4,2 \\
\hline \multicolumn{7}{|c|}{ Distribución según estado civil } \\
\hline Soltero & 7,0 & 23,3 & 6,0 & 33,3 & 13,0 & 27,1 \\
\hline Casado & 19,0 & 63,3 & 11,0 & 61,1 & 30,0 & 62,5 \\
\hline Conviviente & 4,0 & 13,3 & 1,0 & 5,6 & 5,0 & 10,4 \\
\hline
\end{tabular}


Tabla 2.

Caracterización del tiempo en el cargo y antigüedad en el rubro según turno.

\begin{tabular}{lcccccc} 
& \multicolumn{2}{c}{ Turno Rotativo } & \multicolumn{2}{c}{ Turno Diurno } & \multicolumn{2}{c}{ Total } \\
& Promedio & D.E & Promedio & D.E & Promedio & D.E \\
$\begin{array}{lcccccc}\text { Antigüedad } \\
\text { en el rubro }\end{array}$ & 19,8 & 11,1 & 13,4 & 8,0 & 16,0 & 9,7 \\
$\begin{array}{l}\text { Antigüedad } \\
\text { en la empresa }\end{array}$ & 5,7 & 6,7 & 3,1 & 3,6 & 4,1 & 5,1 \\
$\begin{array}{l}\text { D.E: Desviación estándar. } \\
\text { D. }\end{array}$ & & & & &
\end{tabular}

Los trabajadores del turno diurno presentaron, en promedio, una antigüedad en el rubro y en la empresa superior a los del turno rotativo (Tabla 2 ).

\section{Niveles de estrés según turno}

Del personal con turno rotativo, un 20,0\% presentó estrés funcional, y en esa misma proporción, estrés moderado; manifestándose además, en un 10\% de la muestra, estrés agudo. El 22,0\% y $27,8 \%$ del personal que trabaja bajo el sistema de turno diurno presentó estrés funcional y moderado, respectivamente; no manifestándose presencia de estrés agudo en dicho turno (Figura 1).

Figura 1.

Niveles de estrés presentados por los conductores.

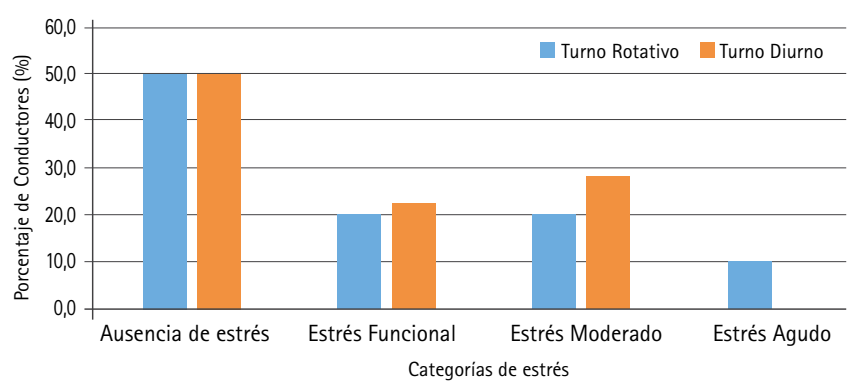

\section{Trastornos del sueńo}

Con respecto al síndrome de insomnio, un 22,2\% de los conductores del turno diurno presentó dicho trastorno, en contraste con el turno rotativo, donde el 60\% de los evaluados manifestó este síndrome en algún nivel, destacándose la presencia de insomnio subclínico en un 46,7\% de la muestra. Para el caso de somnolencia, en ambos turnos se manifestó la presencia de este trastorno, con un 22,3\% y 26,7\% para el turno diurno y rotativo, respectivamente. Respecto a apnea del sueño, un 16,7\% del turno diurno presentó este trastorno en alguno de sus niveles, en tanto para el turno rotativo, este síndrome se manifestó en un 13,3\%. En cuanto al síndrome de piernas inquietas, un 5,6\% del turno diurno y un 6,7\% del turno rotativo presentaron este sindrome, clasificándose a este último como grave (Tabla 3).

Respecto al tiempo que dormían los conductores del turno diurno, más del 75\% lo hacía 7 o más horas diarias. En relación a la presencia de cansancio en su funcionamiento laboral, el 44,4\% de los evaluados manifestó que sí lo presentaba. De las personas que sí presentaron disminución en su funcionamiento laboral (cansancio), el 37,5\% lo percibía durante 2 días. En cuanto a la presencia de alguna enfermedad relacionada con los trastornos del sueño (fibromialgia, cefalea, depresión, arritmias e hipertiroidismos), el $100 \%$ de las personas evaluadas declaró no poseerlas. El 83,3\% de los conductores se dormían durante los primeros 15 minutos; el 11,1\% entre los 15 y 45 minutos y sólo un 5,6\% lo
Tabla 3.

Categorización de Trastornos del sueño.

\begin{tabular}{|c|c|c|c|c|c|c|}
\hline & \multicolumn{2}{|c|}{ Turno Diurno } & \multicolumn{2}{|c|}{ Turno Rotativo } & \multicolumn{2}{|c|}{ Total } \\
\hline & & & & & & $\%$ \\
\hline \multicolumn{7}{|c|}{ Insomnio } \\
\hline Ausencia de insomnio & 14 & 77,8 & 12 & 40,0 & 26 & 54,2 \\
\hline Insomnio subclínico & 4 & 22,2 & 14 & 46,7 & 18 & 37,5 \\
\hline Insomnio moderado & 0 & 0,0 & 4 & 13,3 & 4 & 8,3 \\
\hline Insomnio grave & 0 & 0,0 & 0 & 0,0 & 0 & 0,0 \\
\hline \multicolumn{7}{|c|}{ Somnolencia } \\
\hline Ausencia de somnolencia & 14 & 77,8 & 22 & 73,3 & 36 & 75,0 \\
\hline Somnolencia Ligera & 3 & 16,7 & 6 & 20,0 & 9 & 18,8 \\
\hline Somnolencia Moderada & 1 & 5,6 & 2 & 6,7 & 3 & 6,3 \\
\hline Somnolencia Grave & 0 & 0,0 & 0 & 0,0 & 0 & 0,0 \\
\hline \multicolumn{7}{|c|}{ Apnea del sueño } \\
\hline Ausencia de apnea & 15 & 83,3 & 26 & 86,7 & 41 & 85,4 \\
\hline Existencia de apnea & 2 & 11,1 & 3 & 10,0 & 5 & 10,4 \\
\hline Apnea grave & 1 & 5,6 & 1 & 3,3 & 2 & 4,2 \\
\hline \multicolumn{7}{|c|}{ Sindrome de piernas inquietas } \\
\hline Ausencia del síndrome & 17 & 94,4 & 28 & 93,3 & 45 & 93,8 \\
\hline Existencia del sindrome & 1 & 5,6 & 0 & 0,0 & 1 & 2,1 \\
\hline Síndrome grave & 0 & 0,0 & 2 & 6,7 & 2 & 4,2 \\
\hline
\end{tabular}

realizaba después de los 45 minutos. Respecto a si se despertaban antes de los habitual, el 61,1\% manifestó hacerlo; dentro de esta categoría, el 54,5\% lo hacía media hora antes de lo habitual; un $36,4 \%, 1$ hora antes y un 9,1\%, entre 1 y 2 horas antes (Tabla 4). El 36,7\% de los conductores del turno de noche manifestó dormir 6 horas diarias; para el turno de día, la mayor cantidad de conductores dormía 8 horas diarias, representando un 43,3\% del total de trabajadores. En cuanto a la disminución del funcionamiento laboral, un 50\% del total de trabajadores presentó cansancio o disminución del funcionamiento laboral. Las personas que reconocieron sentirse cansados en sus funciones laborales mencionaron que la mayor extensión de tiempo de esta manifes-

Tabla 4.

Características del sueño de los conductores del turno diurno.

\begin{tabular}{|c|c|c|}
\hline & $\mathrm{N}$ & $\%$ \\
\hline \multicolumn{3}{|c|}{$\mathrm{N}^{\circ}$ de horas que duerme en la noche } \\
\hline 5 horas & 2 & 11,1 \\
\hline 6 horas & 2 & 11,1 \\
\hline 7 horas & 8 & 44,4 \\
\hline 8 horas & 6 & 33,3 \\
\hline \multicolumn{3}{|c|}{ Disminución del funcionamiento laboral (cansancio) } \\
\hline Sí & 8 & 44,4 \\
\hline 1 día & 2 & 25,0 \\
\hline 2 días & 3 & 37,5 \\
\hline 4 dias & 1 & 12,5 \\
\hline 6 dias & 2 & 25,0 \\
\hline No & 10 & 55,6 \\
\hline \multicolumn{3}{|c|}{ Presenta alguna enfermedad relacionada con trastornos del sueño } \\
\hline Sí & 0 & 0 \\
\hline No & 18 & 100 \\
\hline \multicolumn{3}{|c|}{ Tiempo que tarda en dormirse } \\
\hline $0-15 \min$ & 15 & 83,3 \\
\hline $15-45 \mathrm{~min}$ & 2 & 11,1 \\
\hline Más de $45 \mathrm{~min}$ & 1 & 5,6 \\
\hline \multicolumn{3}{|c|}{ Despertaba antes de lo habitual } \\
\hline Sí & 11 & 61,1 \\
\hline $1 / 2$ hora & 6 & 54,5 \\
\hline 1 hora & 4 & 36,4 \\
\hline Entre 1 y 2 horas & 1 & 9,1 \\
\hline Más de 2 horas & 0 & 0,0 \\
\hline No & 7 & 38,9 \\
\hline
\end{tabular}


Tabla 5.

Caracteristicas del sueño de los conductores del turno rotativo.

\begin{tabular}{|c|c|c|}
\hline & $\mathrm{N}$ & $\%$ \\
\hline \multicolumn{3}{|c|}{$\mathrm{N}^{\circ}$ de horas que duerme durante el turno de noche } \\
\hline 4 horas & 1 & 3,3 \\
\hline 5 horas & 3 & 10,0 \\
\hline 6 horas & 11 & 36,7 \\
\hline 7 horas & 7 & 23,3 \\
\hline 8 horas & 6 & 20,0 \\
\hline 9 horas & 2 & 6,7 \\
\hline \multicolumn{3}{|c|}{$\mathrm{N}^{\circ}$ de horas que duerme durante el turno de día } \\
\hline 5 horas & 3 & 10,0 \\
\hline 6 horas & 10 & 33,3 \\
\hline 7 horas & 3 & 10,0 \\
\hline 8 horas & 13 & 43,3 \\
\hline 11 horas & 1 & 3,3 \\
\hline \multicolumn{3}{|c|}{ Disminución del funcionamiento laboral (cansancio) } \\
\hline Si & 15 & 50,0 \\
\hline 1 día & 3 & 20,0 \\
\hline 2 dias & 5 & 33,3 \\
\hline 3 días & 3 & 20,0 \\
\hline 4 días & 2 & 13,3 \\
\hline 6 días & 2 & 13,3 \\
\hline No & 15 & 50,0 \\
\hline \multicolumn{3}{|c|}{ Tiempo que tarda en dormirse } \\
\hline $0-15 \mathrm{~min}$. & 11 & 36,7 \\
\hline $15-45 \mathrm{~min}$. & 15 & 50,0 \\
\hline Más de $45 \mathrm{~min}$. & 4 & 13,3 \\
\hline \multicolumn{3}{|c|}{ Presenta alguna enfermedad relacionada con trastornos del sueño } \\
\hline Si & 1 & 3,3 \\
\hline No & 29 & 96,7 \\
\hline \multicolumn{3}{|c|}{ Días en los que comienza a adaptarse a su turno } \\
\hline 1er dia & 8 & 26,7 \\
\hline 2do día & 16 & 53,3 \\
\hline 3er día & 4 & 13,3 \\
\hline 4to día & 1 & 3,3 \\
\hline 5to día & 1 & 3,3 \\
\hline \multicolumn{3}{|c|}{ Turno que le produce mayor insomnio } \\
\hline Turno de noche & 15 & 50,0 \\
\hline Turno de día & 3 & 10,0 \\
\hline Ninguno & 12 & 40,0 \\
\hline \multicolumn{3}{|c|}{ Turno que le produce mayor somnolencia } \\
\hline Turno de noche & 19 & 63,3 \\
\hline Turno de día & 2 & 6,7 \\
\hline Ninguno & 9 & 30,0 \\
\hline
\end{tabular}

tación fue de 2 días, con un 33,3\%; en cambio, un 50\% del total de trabajadores manifestó no sentirse cansado. El tiempo que con mayor frecuencia tardaban en dormirse los conductores evaluados, estaba entre los 15 y 45 minutos, representado por un 50\% de la muestra total. Un $36,7 \%$ se dormía durante los primeros 15 minutos y un 13,3\% lo hacía después de 45 minutos. Con respecto a la presencia de alguna enfermedad relacionada con los trastornos del sueño, solo una persona presentó una sintomatología relacionada con éstos (cefalea). La adaptación de los turnos también influye en el desarrollo del sueño: el 53,3\% de las personas evaluadas manifestó adaptarse a los 2 días de haber comenzado su turno. En cuanto a los turnos que producen mayor insomnio y somnolencia, los datos muestran que el turno de noche generaba mayor insomnio y somnolencia, en un 50\% y 63,3\% de la muestra, respectivamente (Tabla 5).

\section{Riesgo psicosocial en los turnos evaluados}

En las figuras 2 y 3 se muestran los niveles de exposición a riesgo psicosocial en cada turno evaluado. Para ambos tipos de turnos (diurno y rotativo), las dimensiones doble presencia y compensa-
Figura 2.

Semáforo de riesgo psicosocial del turno diurno.

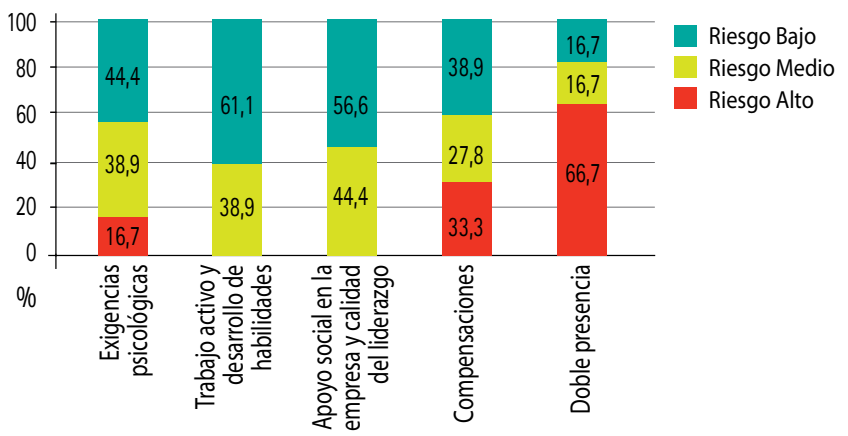

ciones concentran los mayores porcentajes asociados a un riesgo alto, con un 33,3\% y un 66,7\%, respectivamente, clasificándose sólo como riesgo alto la dimensión doble presencia para el turno diurno (color rojo) (Figura 2).

El turno diurno presentó las dimensiones 2 (Trabajo activo y desarrollo de habilidades) y 3 (Apoyo social en la empresa y calidad del liderazgo) con un 61,1\% y 55,6\% de riesgo bajo, respectivamente, constituyendo la situación más favorable para la salud de los trabajadores. Aunque en estas mismas dimensiones, el riesgo medio sigue latente, con un 38,9\% y 44,4\% respectivamente, sumándose las dimensiones 1 (Exigencias psicológicas) y 4 (Compensaciones), con un 38,9\% y 27,8\%, respectivamente (Figura 2). Asimismo, en el turno rotativo, al igual que en el turno diurno, la condición más favorable para la salud de los conductores son las dimensiones 2 y 3, con un 56,7\% y 56,7\% de nivel de exposición a riesgo bajo. Para el turno rotativo, el riesgo medio se encuentra latente en las 5 dimensiones, predominando la dimensión 1 , que posee un 43,3\%; la segunda con un 40\%; 3 y 4 poseen un 33,3\%; y, por último, la dimensión 5, con un 36,7\% (Figura 3). El turno diurno presentó una dimensión en riesgo alto, con un 66,7\% (Doble presencia) (Figura 2), clasificándose, de acuerdo al Protocolo de Vigilancia de Riesgos Psicosociales ${ }^{24}$, en la categoría de "Riesgo alto/Nivel 1". Para el turno rotativo, ninguna dimensión fue categorizada en riesgo alto ni en riesgo moderado, por lo que este turno se clasifica como "Riesgo Bajo" (Figura 3).

\section{Impacto de los turnos sobre los trabajadores}

Un 66,7\% y 96,7\% de los trabajadores de los turnos diurno y rotativo, respectivamente, fueron clasificados en riesgo medio, en cuanto al impacto generado por el tipo de turno. Esta situación puede explicarse, ya que el segundo turno en cuestión trabaja una

Figura 3.

Semáforo de riesgo psicosocial del turno rotativo.

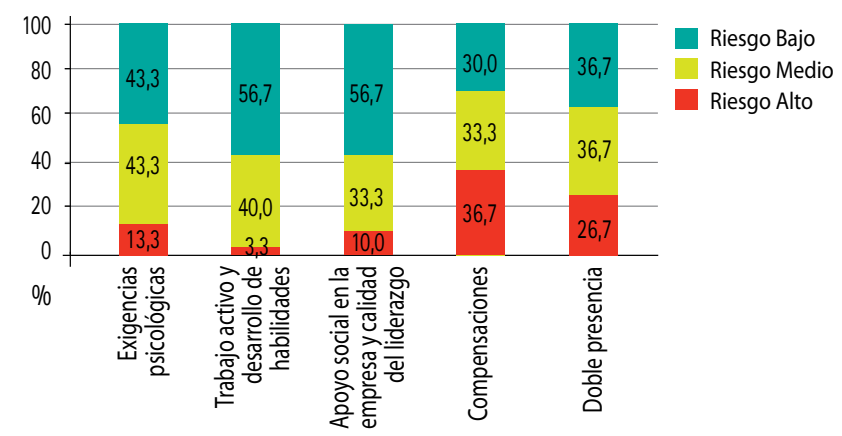


Tabla 6.

Impacto de los turnos en conductores del turno rotativo y diurno.

\begin{tabular}{lcccc} 
& \multicolumn{2}{c}{ Turno diurno } & \multicolumn{2}{c}{ Turno } \\
Nivel de riesgotivo \\
Bajo & N & $\%$ & N & $\%$ \\
Medio & 6 & 33,3 & 1 & 3,3 \\
Alto & 12 & 66,7 & 29 & 96,7 \\
& 0 & 0,0 & 0 & 0,0
\end{tabular}

Tabla 7.

Accidentabilidad según tipo de turno.

\begin{tabular}{lcccc}
\multicolumn{4}{c}{} & \multicolumn{2}{c}{$N^{\circ}$ de accidentes } & & \\
& Turno Diurno & \multicolumn{2}{c}{ Turno Rotativo } \\
Si & N & $\%$ & N & $\%$ \\
No & 3 & 16,7 & 4 & 13,3 \\
& 15 & 83,3 & 26 & 86,7
\end{tabular}

secuencia de una semana de día y una de noche, por lo que el impacto que se genera en la salud y vida social del conductor se identifica con mayor facilidad. Es importante destacar que no se clasificaron trabajadores con un nivel de riesgo alto de impacto a causa del sistema de turnos (Tabla 6).

\section{Accidentabilidad según tipo de turno}

De ambos turnos evaluados, el sistema diurno presentó el mayor porcentaje de conductores que manifestó haber sufrido algún accidente (16,7\%) (Tabla 7).

\section{Relación de la accidentabilidad con las variables estrés, trastornos del sueńo, riesgos psicosociales e impacto de los turnos}

Para el turno diurno, las variables que se correlacionaron significativamente con la accidentabilidad fueron estrés y apoyo social (dimensión 3 de riesgos psicosociales); en contraste, el turno rotativo, presentó solo una variable relacionada en forma significativa con la accidentabilidad, compensaciones (dimensión 4 de riesgos psicosociales). Para el total de trabajadores, se determinaron correlaciones significativas entre la accidentabilidad y apoyo social y compensaciones (Tabla 8).

\section{DISCUSIÓN}

López y Manriquez ${ }^{33}$, determinaron la existencia de una estrecha relación entre el trabajo en turnos rotativos y estrés laboral. El constante proceso de adaptación al que se ven sometidos los trabajadores que desempeñan labores en turnos rotativos, supone una fuente de estrés para ellos, situación que se contrapone con los niveles obtenidos en el presente estudio en ambos turnos, en donde el 50\% de los trabajadores no presentó estrés. El turno rotativo presentó este sintoma en sus tres niveles (funcional, moderado y agudo); por su parte, el turno diurno también presentó el 50\% de sus conductores con estrés, pero distribuido solo en las categorías funcional y moderado (Figura 1).

Según Osca, López-Araujo, Bardera, Urien, Díez y Rubio ${ }^{34}$, los síntomas a los que menor importancia se les da, con respecto a la generación de accidentes, son los relacionados con el área psicológica. Estos pueden actuar como mediadores entre los estresores del trabajo y los accidentes, lo que se podría explicar a partir de la Teoría de modificación del estrés, ya que aspectos como la preocupación o la ansiedad pueden hacer que se preste menos
Tabla 8.

Correlaciones entre accidentabilidad y variables de estrés, trastornos del sueño, riesgo psicosocial e impacto de los turnos.

\begin{tabular}{|c|c|c|c|c|}
\hline \multicolumn{2}{|c|}{ Variables evaluadas } & \multirow{2}{*}{$\begin{array}{c}\text { Turno Diurno } \\
\text { Accidentes } \\
0,55^{*}\end{array}$} & \multirow{2}{*}{$\begin{array}{c}\text { Turno Rotativo } \\
\text { Accidentes } \\
0,12\end{array}$} & \multirow{2}{*}{$\begin{array}{c}\text { Total Trabajadores } \\
\text { Accidentes } \\
0,28\end{array}$} \\
\hline Estrés & Estrés & & & \\
\hline \multirow{4}{*}{$\begin{array}{l}\text { Trastornos } \\
\text { del sueño }\end{array}$} & Insomnio & 0,20 & 0,14 & 0,13 \\
\hline & Somnolencia & $-0,07$ & $-0,05$ & $-0,05$ \\
\hline & Apnea & $-0,38$ & 0,12 & $-0,09$ \\
\hline & Piernas inquietas & $-0,11$ & 0,36 & 0,20 \\
\hline $\begin{array}{l}\text { Impacto } \\
\text { de turnos }\end{array}$ & $\begin{array}{l}\text { Impacto } \\
\text { de turnos }\end{array}$ & 0,36 & 0,33 & 0,14 \\
\hline \multirow{5}{*}{$\begin{array}{l}\text { Riesgo } \\
\text { psicosocial }\end{array}$} & $\begin{array}{l}\text { Exigencias } \\
\text { psicológicas }\end{array}$ & 0,30 & 0,23 & 0,23 \\
\hline & Trabajo activo & 0,16 & $-0,02$ & 0,05 \\
\hline & Apoyo social & $0,57^{*}$ & 0,23 & $0,38^{*}$ \\
\hline & Compensaciones & 0,25 & $0,42^{*}$ & $0,35^{*}$ \\
\hline & Doble presencia & 0,28 & 0,31 & 0,28 \\
\hline
\end{tabular}

atención al trabajo, aumentando la probabilidad de accidentarse y/o de tener incidentes.

El turno rotativo mostró resultados similares en cuanto a estrés a los obtenidos por otros autores, tales como Castillo, Torres, Ahumada, Cárdenas y Licona ${ }^{35}$, quienes determinaron que las personas que se desempeñan en turnos rotativos presentaban niveles críticos de estrés, comparados con los niveles de estrés de los trabajadores del turno diurno, resultado que coincide con lo obtenido en el presente estudio, donde el turno rotativo presentó estrés en su categoría más crítica (estrés agudo).

Los resultados obtenidos en ambos turnos concuerdan con lo determinado por Rodríguez ${ }^{36}$, quien estableció que los trabajadores sometidos a trabajos con turnos rotativos se encuentran más predispuestos a sufrir trastornos del sueño, fatiga e irritabilidad. Esto explicaría los porcentajes obtenidos en la evaluación de insomnio en el turno rotativo, donde un 60\% de los conductores presentó este síndrome en alguno de sus niveles. Diversas investigaciones evidencian que el turno de noche afecta constantemente el sueño. Fernández-Montalvo y Piñol ${ }^{4}$, establecieron que los problemas de sueño se presentan especialmente en trabajadores que tienen turnos rotativos ya que, al encontrarse en turno de noche, se ven forzados a dormir cuando el organismo se inclina a estar despierto $\mathrm{y}$, por el contrario, a permanecer alerta cuando el ciclo del organismo está predispuesto al sueño. Lo anterior explicaría los trastornos del sueño manifestados en los turnos bajo estudio: insomnio, somnolencia y síndrome de piernas inquietas, presentando el turno rotativo una mayor cantidad de casos, en comparación con el turno diurno. Contrario a lo mencionado anteriormente por dichos autores, el sindrome de apnea del sueño en el turno diurno presentó una mayor prevalencia que en el turno rotativo. Este último síndrome podría tener relación con otros aspectos de los trabajadores, como la composición corporal, el peso, el índice masa grasa, los cuales pueden influir en la generación de este trastorno. ${ }^{16,37,38}$

Lac y Chamoux ${ }^{39}$ determinaron que la falta de sueño asociado al trabajo por turnos puede generar importantes elevaciones de cortisol en la sangre, lo que es un potente indicador de haber estado estresado durante bastante tiempo, por lo que los resultados obtenidos respecto de la presencia de trastornos del sueño en cualquiera de sus categorías pudo haber influenciado la evaluación de estrés en los conductores, independiente del tipo de turno.

Los cambios de horario en los turnos rotativos hacen a los trabajadores más susceptibles, en términos generales, a riesgos poten- 
ciales -como generación de micro-sueños y déficit de atención a la hora de conducir- que aquellos que trabajan en turnos diurnos, produciéndose una frecuente reducción de la capacidad de atención desde la primera hora del turno, disminuyendo la capacidad de concentración y de la memoria. ${ }^{36}$ Esto explicaría los resultados obtenidos en cuanto a disminución del funcionamiento laboral, dado que el turno rotativo presentó un 50\% del total de conductores que reconocía sentirse cansado, y un $44 \%$ para el caso del turno diurno.

En lo que respecta a la duración del sueño, al trabajar en turno rotativo, se produce una reducción notable del número de horas destinadas a dormir. Fernández-Montalvo y $\mathrm{Piñol}^{4}$ afirman que la duración del sueño es significativamente menor en los trabajadores que se encuentran en turno de noche, en comparación con los del turno diurno; en contraste con lo mencionado anteriormente, el mayor porcentaje de trabajadores del turno rotativo manifestó dormir 6 horas cuando está en turno nocturno, lo que representa un 36,7\% del total de trabajadores del turno rotativo; en cambio, en el turno diurno, un 43,3\% del total de trabajadores lo hacía 7 horas. Esto puede deberse a la variabilidad en cuanto al horario de inicio de cada turno, pudiendo fluctuar entre las 5:30 am y las 9:00 am. Autores como Miró, Cano-Lozano y BuelaCasal $^{40}$ indican que los períodos cortos de sueño tienen un impacto negativo en el metabolismo de los carbohidratos y en la función endocrina. Ambos factores están considerados partes fundamentales del proceso normal de envejecimiento, por lo que si persistiera en el organismo el hábito de acortar los períodos de sueño, se incrementaría la gravedad del trastorno crónico asociado a la edad (envejecimiento acelerado). Los trabajadores que duermen menos de 5 horas pueden sufrir cambios notables en la concentración de glucosa, tirotropina y melatonina. La tirotropina es una hormona estimulante de la tiroides, que se rige por el ciclo del ritmo circadiano, liberándose en mayor cantidad durante el día y descendiendo sus niveles al dormir; pero al privar de sueño al organismo o alterar el ciclo sueño-vigilia, se genera un $200 \%$ de esta hormona. Por su parte, la melatonina favorece la generación del sueño de acuerdo a la exposición a luz, su liberación nocturna se inhibe por la luz, influyendo además, en el ritmo de la temperatura corporal, secreción de cortisol (secreción generada por estrés) y sobre la secreción interna de la misma hormona, pudiéndose generar enfermedades relacionadas con estas hormonas (Diabetes mellitus, obesidad, hipotiroidismo e hipertiroidismo). ${ }^{41}$

Los trabajadores sometidos a frecuentes rotaciones de turnos son quienes presentan mayores dificultades de adaptación, ya que fuerzan el sueño y la vigilia en función de un ritmo circadiano aberrante, lo que imposibilita cualquier intento de adaptación, por lo que los trabajadores del turno rotativo evaluado deberían comenzar a adaptarse a su turno, al término del mismo, lo que produciría la alteración del ritmo circadiano. ${ }^{4} \mathrm{~A}$ pesar de lo anterior, el 53,3\% de los conductores evaluados respondieron que se adaptaban al segundo día de su turno, y sólo un 6,6\% manifestó $\mathrm{su}$ adaptación al $4^{\circ}$ y $5^{\circ}$ día (Tabla 5). Las contradicciones obtenidas con respecto a los resultados del presente estudio pueden ser influenciadas por la percepción de adaptación que tiene cada conductor, la cual no es directamente comparable respecto a la alteración del ritmo circadiano, determinado clínicamente. Fernández-Montalvo y $\mathrm{Piñol}^{4}$ demostraron que el turno que produce mayor somnolencia en los trabajadores es el turno de noche, ya que dormitan durante el período en que desearian estar despiertos, como exige su horario laboral. Esto respalda los resultados obtenidos en la presente investigación, en donde el 63,3\% de los trabajadores aseguró que el turno que le presentaba mayor somnolencia correspondía al turno de noche (Tabla 5). Cabe mencionar que cada persona tiene diferentes respuestas fisiológicas con respecto al sueño, ya que para algunos es más fácil sucumbir al sueño durante la noche, generándose micro-sueños.

Tovalín, Rodríguez y Ortega ${ }^{42}$ determinaron que los trabajadores en turno rotativo presentan un riesgo de 7 a 14 veces mayor que los trabajadores de turno fijo de tener alteraciones del sueño tales como: despertarse durante el sueño, tener somnolencia durante el período de vigilia y sentirse cansados durante el día. En el presente estudio, ambos turnos evaluados presentaron trabajadores que conciliaban el sueño dentro de los primeros 15 minutos; en cambio, el turno rotativo presentó casos en donde el tiempo para conciliar el sueño fluctuó entre los 15 y 45 minutos, y un porcentaje inferior lo realizaba en un tiempo mayor a 45 minutos (Tabla 5). Esto se puede explicar debido al funcionamiento normal del ritmo circadiano, por lo que al estar despierto en el momento que el cuerpo debe dormir se altera el ciclo de sueño-vigilia, repercutiendo en el momento en que el trabajador desea dormir. En el presente estudio no se especificó en qué turno le costaba más conciliar el sueño a los trabajadores, por lo que es interesante estudiar en qué momento del ciclo sueño-vigilia se altera con mayor frecuencia el ritmo circadiano, generando un obstáculo para conciliar el sueño. Variados estudios respaldan que los trabajadores por turnos muestran mayores dificultades para conciliar el sueño o demoran más tiempo en quedarse dormidos que los que no trabajan por turnos; tal dificultad ha sido informada por Marquie y Foret ${ }^{43}$, Akerstend ${ }^{13}$ y Medina y Sierra. ${ }^{44}$

López y Manríquez ${ }^{33}$ determinaron que las condiciones en las que se desempeña un puesto de trabajo influyen de forma significativa en la salud mental del trabajador, donde la oportunidad de control, la adecuación entre las exigencias del puesto y las capacidades de la persona que lo desempeña, las relaciones interpersonales, salario y seguridad física son, entre otros, aspectos importantes que ejercen una gran influencia sobre el bienestar psicológico. En los resultados obtenidos de la evaluación de riesgos psicosociales, se destaca un descontento con la dimensión compensaciones, manifestándose en color rojo para ambos turnos, con un 33,3\% para el turno diurno y 36,7\% para el rotativo, aunque estos porcentajes no clasifican a los turnos con riesgo alto en esta dimensión. Respecto a la dimensión doble presencia, la cual se relaciona con el hogar y la organización, el turno diurno presentó un 66,7\% en riesgo alto; en cambio, el turno rotativo, que debiese ser el más afectado por la forma de trabajo en la que se encuentra, solo presentó un $26,7 \%$ de riesgo en este nivel (Figuras 2 y 3). Los resultados del turno diurno podrian ser explicados por la duración del turno ya que, generalmente, el turno comienza temprano (entre 6 y 8 de la mañana) y, dada la imposibilidad de cambio, no les permite programar sus actividades cotidianas; en cambio, el turno rotativo tiene la alternativa de realizar sus actividades del hogar cada una semana, aunque implique dormir menos horas. Al respecto, Miró, Cano-Lozano y BuelaCasal $^{40}$ mencionan que los trabajadores consideran que las características del trabajo nocturno no sólo alteran su sueño, sino que también crea dificultades en su vida familiar, restringe su vida social, reduce sus actividades de ocio y afecta su salud. Esto explicaría los resultados obtenidos en la evaluación de riesgo psicosocial del turno rotativo, en donde se muestran porcentajes en 
riesgo alto para la dimensión doble presencia (26,7\%) (Figura 3). El impacto del trabajo a turnos puede no afectar de la misma manera a los trabajadores. Hay quienes se adaptan a este tipo de trabajo, mientras que otros presentan problemas más graves, que los llevan a solicitar su cambio de turno o a renunciar al trabajo. ${ }^{45}$ El resultado obtenido en la evaluación del impacto de los turnos en los trabajadores del turno rotativo puede explicarse debido a que éstos se encuentran en un constante cambio de horarios, repercutiendo tanto en su vida familiar como en su salud; en cambio, el turno diurno no debería presentar trabajadores con riesgo medio, ya que su turno se mantiene en el tiempo, aunque esto puede estar relacionado con los resultados entregados por el protocolo de riesgos psicosociales, en donde el turno diurno obtuvo la dimensión doble presencia en riesgo alto. En consecuencia, los resultados obtenidos para el turno diurno demuestran que éstos se ven influenciados por el tipo de turno en el que realizan su trabajo, ya que afecta en el desarrollo normal de su vida cotidiana al no disponer de tiempo para realizar sus quehaceres cotidianos. Kageyama, Nishikido, Kobayashi, Oga y Kawashima $^{46}$ indican que los efectos nocivos de los sistemas de trabajo se pueden ver intensificados si se presenta una serie de factores de riesgo como, por ejemplo, tener más de 45 años, presentar un alto indice de masa corporal, no hacer ejercicio y disponer de poco apoyo social, por lo que explicaría los resultados obtenidos en la evaluación del impacto de los turnos en los trabajadores.

Rodríguez $^{36}$ y Córdova ${ }^{47}$ establecieron que los conductores que desempeñan labores en turnos nocturnos son más propensos a sufrir accidentes, ya que el desarrollo del turno se produce durante el punto más bajo de actividad del ritmo circadiano. Los resultados obtenidos para esta variable pudieron ser influenciados por otros factores, como la desconcentración a la hora de realizar su trabajo, ya que los conductores del turno diurno presentaron estrés en su categoría de moderado (Figura 1); además, de un porcentaje mayor en riesgo alto para la dimensión de exigencias psicológicas (Figura 2).

Sólo el turno diurno presentó una relación significativa entre accidentabilidad y estrés, ya que el 50\% de los conductores evaluados de dicho turno presentó estrés en las categorias funcional y moderado (Figura 1). Respecto a la asociación entre accidentabilidad y apoyo social, ésta contrasta con los resultados obtenidos en la evaluación de riesgos psicosociales (Figura 2), ya que solo un 44,4\% presentó riesgo medio, no existiendo casos con riesgo alto. Esta relación indicaría que a menor apoyo social de la empresa hacia los trabajadores mayor será la probabilidad de que los conductores tengan accidentes. El apoyo social depende de cómo lo perciba el trabajador, ya que la empresa puede brindar las mejores condiciones, pero si el trabajador no tiene la predisposición a aceptarlas, la percepción que el trabajador tiene de la empresa no se modificará.

Para el turno rotativo, la correlación entre accidentabilidad y compensaciones se explica basado en los resultados obtenidos de la evaluación de riesgos psicosociales del turno, en donde se obtuvo para la dimensión de compensaciones un 70\% del total de conductores del turno clasificados en riesgo medio $(33,3 \%)$ y alto (36,7\%) (Figura 3).

En conclusión, la evaluación de estrés realizada a los conductores determinó, para el turno rotativo, la presencia de este síndrome en sus niveles funcional, moderado y agudo. Por su parte, el turno diurno solo presentó conductores con niveles de estrés funcional y moderado. Respecto a la presencia de trastornos del sueño, el turno rotativo presentó una mayor cantidad de casos que el turno diurno para los sindromes: insomnio, somnolencia y sindrome de piernas inquietas; por su parte, el turno diurno presentó un mayor número de casos para el sindrome de apnea del sueño. La evaluación de riesgos psicosociales clasificó al turno diurno con Riesgo Alto/ Nivel 1, y al turno rotativo, en Riesgo Bajo. La medición del impacto de los turnos en los trabajadores clasificó al turno rotativo y diurno en riesgo medio, con un $96,7 \%$ y un $66,7 \%$ de la muestra, respectivamente. El turno que presentó mayor cantidad de accidentes correspondió al turno diurno, con un 16,7\%, en comparación al turno rotativo (13,3\%). El análisis de correlación determinó que existe relación entre accidentabilidad y las variables evaluadas, presentándose correlaciones significativas para el turno diurno, entre accidentabilidad, estrés y apoyo social (dimensión 3 de riesgos psicosociales). Por su parte, el turno rotativo presentó correlación significativa sólo entre accidentabilidad y compensaciones (dimensión 4 de riesgos psicosociales). 
1. Morales G. En el camino: Los conductores de camiones de carga y sus condiciones laborales [en línea]. Santiago: Dirección del Trabajo; 2011. (Cuaderno de investigación; 41) [citado mar 2017]. Disponible en: http://www.dt.gob. cl/1601/articles-100038_recurso_1.pdf

2. Garbarino S, Nobili L, Beelke M. Somnolencia diurna, factor relevante en accidentes vehiculares; resumen del artículo (en línea). Sleep. 2001[citado mar 2017]; 24(2):203-206 Disponible en: http://www.institutodelsueno.cl/faltasomnolencia-diurna-factor-relevante-en-accidentes-vehiculares/

3. Comisión Nacional de Seguridad de Tránsito. Diagnóstico de accidentes de tránsito en la región del BíoBío. Informe anual de tránsito 2015 [en línea]. Santiago: CONASET; 2016 [citado mar 2016]. Disponible en: http://www. conaset.cl/wp-content/uploads/2016/07/NIII-region-2015.pdf

4. Fernández-Montalvo J, Piñol E. Horario laboral y Salud: Consecuencias Psicológicas de los turnos de trabajo. Rev Psicopatol Psicol Clín. 2000; 5(3):207-222.

5. Echeverría M, López D, Santibáñez I, Vega H. Flexibilidad laboral en Chile: Las empresas y las personas. Santiago: Dirección del Trabajo; 2004. (Cuaderno de investigación; 22).

6. Chile. Ministerio del Trabajo y Previsión Social. Guía Técnica para la Evaluación de Trabajo Pesado [en línea]. Santiago: Ministerio del Trabajo y Previsión Social; 2010 [citado mar 2017]. Disponible en: http://www.spensiones.cl/portal/institucional/578/articles-8418_guia_tecnica.pdf

7. Maslach $\mathrm{C}$, Jackson SE. The measurement of experienced burnout. J Occup Behav. 1981; 2:99-113.

8. Harrington J. Health effects of shiftwork and extended hours of work. Occup Environ Med. 2001; 58:68-78.

9. Bohle P, Tilley AJ. The impact of night work on psychological wellbeing. Ergonomics. 1989; 32:1089-1099.

10. Akerstend T. Psychological and physiological effects of shiftwork. Scand J Work Env Hea. 1990;16:67-73.

11. Colligan $M$, Rosa R. Shiftwork effects on social and family life. Occup Med-State Art. 1990; 5(2):315-322.

12. Morshead D. Stress and Shiftwork. Occup Health Safe. 2002;71(4): 36-38.

13. Akerstend T. Shift work and disturbed sleep/wakefulness .0ccup Med. 2003; 53:89-94.

14. Gállego J, Toledo J, Urrestarazu E, Iriarte J. Clasificación de los trastornos del sueño. An Sist Sanit Navar. 2007; 30(1):19-36.

15. Contreras A. Insomnio: generalidades y alternativas terapéuticas de última generación. Rev Méd Clín Condes. 2013; 24(3):433-441.

16. Olivi H. Apnea del sueño: cuadro clínico y estudio diagnóstico. Rev Méd Clín Condes. 2013; 24(3):359-373.

17. American Academy of Sleep Medicine. The International Classification of Sleep Disorders. Diagnostic and Coding Manual [on line]. Westchester: American Academy of Sleep Medicine; 2001 [cited mar 2017]. Disponible en: http://www. esst.org/adds/ICSD.pdf

18. Healy $D$, Waterhouse J. Reactive rhythms and endogenousclocks. Psychol Med. $1991 ; 21: 557-564$.

19. Moreno B, Baez C. Factores y riesgos psicosociales, formas, consecuencias, medidas y buenas prácticas. Madrid; INSHT; 2011; 188 p [citado mar 2017]. Disponible en: http://www.insht.es/InshtWeb/Contenidos/Documentacion/ PUBLICACIONES\%2OPROFESIONALES/factores\%20riesgos\%20psico.pdf

20. Gimeno D. Los factores de riesgo laboral de naturaleza psicosocial y su prevención. Arch Prev Riesgos Labor. 2004; 7(3):119-120.

21. Cohen S, Kamarck T, Mermelstein R. A Global Measure of Perceived Stress. J Health Soc Behav. 1983; 24:385-396.

22. Meyer $A$, Ramírez $L$, Pérez $C$. Percepción de estrés en estudiantes chilenos de Medicina y Enfermeria. Rev Educ Cienc Salud. 2013; 10(2):79-85.

23. Tapia D, Cruz C, Gallardo L, Dasso M. Adaptación de la Escala de percepción Global de estrés en estudiantes adultos de escasos recursos en Santiago, Chile. Admin Sanit. 2007; 24(1-2):109-119.
24. Chile. Ministerio de Salud. Protocolo de vigilancia de riesgos psicosociales en el trabajo. Santiago: MINSAL; 2013 [citado mar 2017]. Disponible en: http:// web.minsal.cl/portal/url/item/e039772356757886e040010165014a72.pdf

25. Gissel A, Knauth P. Assessment of shift in German industry and service sector: A computer application of the Besiak procedure. Int J Ind Ergonom. 1998; 21: 233-242.

26. Célyne $H$, Valliéres $A$, Morin $C$. Validation of the insomnia Severity Index as an outcome measure for insomnia research. Québec: École de Psychologie and Centre d'Etude des troubles du Sommeil-Université Laval; 1999.

27. Johns M. A new method for measuring daytime sleepiness: The Epworth Sleepiness Scale. Sleep. 1991;14(6):540-545.

28. Buysse D, Reynolds C, Monk T, Berman S, Kupfer D. The Pittsburg Sleep Quality Index: A New Instrument for Psychiatric Practice and Research. Psychiatr. 1989; 28:193-213.

29. Bobes J, González G, Saiz P, Bascarán M, Iglesias C, Fernández, J. Propiedades psicométricas del cuestionario Oviedo del sueño. Psicothema. 2000; 12(1):107112.

30. Vicéns J, Medina E. Análisis de datos cualitativos; Tablas de contingencia. [Madrid: UAM]; 2005 [citado mar 2017]. Disponible en: https://www.uam.es/ personal_pdi/economicas/eva/pdf/tab_conting.pdf

31. Mendenhall W, Beaver RJ, Beaver BM. Introducción a la probabilidad y estadistica. 13a. ed. México DF: Cengage Learning; 2010. 744 p.

32. Peña D. Fundamentos de Estadística. Madrid: Alianza; 2001. $688 p$

33. López $\mathrm{P}$, Manrique M. Riesgos psicosociales en trabajadores de hospital psiquiatrico. Valladolid: Universidad de Valladolid; 2013.

34. Osca A, López-Araujo B, Bardera P, Urien B, Díez V, Rubio C. Riesgos psicosociales y accidentabilidad laboral: Investigación y propuestas de actuación. Papeles del psicólogo 2014; 35(2):138-143.

35. Castillo I, Torres N, Ahumada A, Cárdenas K, Licona S. Labor stress in nursing and associate factors; Cartagena (Colombia). Salud (Barranquilla). 2014; 30(1):34-43.

36. Rodríguez C. Efectos de los turnos de trabajo y el ritmo circadiano. Salud, Trabajo y Ambiente-CCS. 2005; 46.

37. Hernández $M$, Parra $L$, Pérez $A$. Revisión bibliográfica exploratoria sobre sindrome de apnea obstructiva del sueño y conducción profesional. Med Segur Trab. 2012; 59(227):148-167.

38. Xiang G, Fang Y, Guo F, Hanowski R. The influence of daily sleep patterns of commercial truck drivers on driving performance. Accident Anal Prev. 2016; 91:55-63.

39. Lac $G$, Chamoux A. Elevated salivary cortisol levels as a result of sleep deprivation in a shift worker. Occup Med. 2003; 53(2):143-145.

40. Miró E, Cano-Lozano M, Buela-Casal G. Sueño y calidad de vida. Rev Colomb Psicol. 2005; 14(1):11-27.

41. Sierra L. Trabajo en turnos, privación del sueño y sus consecuencias clínicas y médico-legales. Rev Méd Clin Condes. 2003; 24(3):443-451.

42. Tovalin $\mathrm{H}$, Rodríguez $\mathrm{M}$, Ortega $\mathrm{M}$. Rotación de turnos, fatiga y trastornos del sueño en un grupo de trabajadores industriales. Rev Cubana Salud Trabajo. 2015; 6(1):27-31.

43. Marquie J, Foret J. Sleep, age and shiftwork experience. J Sleep Res. 1999; 8(4):297-304.

44. Medina A, Sierra J. Influencia del trabajo por turnos en el estado emocional y en la calidad del sueño. Psicología y Salud. 2004; 14(2):147-154.

45. Tellez A, Villegas D, Juárez D, Segura L, Fuentes $L$. Trastornos y calidad de sueño en trabajadores industriales de turno rotatorio y turno fijo. Univ Psychol. 2015; 14(2):695-706

46. Kageyama T, Nishikido N, Kobayashi T, Oga J, Kawashima M. Cross-sectional survey on risk factors for insomnia in Japanese female hospital nurses working rapidly rotating shift systems. J Human Ergol. 2001; 30(1-2):149-154.

47. Córdova V. Patrón de accidentabilidad en el trabajo en turnos de 7.707 empresas: Experiencia ACHS. Cienc Trab. 2004; 6(13):126-131. 